\title{
A Silicon UCN Detector With Large Area and With Analysis of UCN Polarization
}

\begin{tabular}{lll}
\hline Volume 110 & Number 3 & May-June 2005 \\
\hline
\end{tabular}

\section{Lasakov, A. Serebrov, A. Khusainov, A. Pustovoit, Yu. Borisov, and A. Fomin}

St. Petersburg Nuclear Physics Institute, Gatchina, Russia

\section{P. Geltenbort}

Institut Max von Laue - Paul Langevin, Grenoble, France

\section{O. Kon'kov}

Ioffe Physical Technical Institute, St. Petersburg, Russia

and

I. Kotina, A. Shablii, V. Solovei, and A. Vasiliev

St. Petersburg Nuclear Physics Institute, Gatchina, Russia

\begin{abstract}
A silicon ultracold neutron (UCN) detector with an area of $45 \mathrm{~cm}^{2}$ and with a ${ }^{6} \mathrm{LiF}$ converter is developed at St. Petersburg Nuclear Physics Institute (PNPI). The spectral efficiency of the silicon UCN detector was measured by means of a gravitational spectrometer at Institut Max von Laue - Paul Langevin (ILL). The sandwich-type detector from two silicon plates with a ${ }^{6} \mathrm{LiF}$ converter placed between them was also studied. Using this type of technology the UCN detector with analysis of polarization was developed and tested. The analyzing power of this detector assembly reaches up $75 \%$ for the main part of UCN spectrum. This UCN detector with analysis of UCN polarization can be used in the new electric dipole moment (EDM) spectrometer.
\end{abstract}

Accepted: August 11, 2004

Available online: http://www.nist.gov/jres

\section{A Si UCN Detector With Large Area and Measurement of Its Energy Dependent Efficiency}

For the production of the Si UCN detector with large area we used a Si wafer of $78 \mathrm{~mm}$ diameter. The area of the detector $\left(48 \mathrm{~cm}^{2}\right)$ was divided into four parts to decrease the capacity of the detector. It allows one to keep the amplitude of the signal at a sufficiently high level with respect to detector noise. The surface of the detector was coated with ${ }^{6} \mathrm{LiF}\left(80 \%{ }^{6} \mathrm{Li}\right.$ enrichment) with a thickness of $0.6 \mathrm{mg} / \mathrm{cm}^{2}$ to $0.8 \mathrm{mg} / \mathrm{cm}^{2}$. The energy dependence of the registration efficiency was measured using the gravitational spectrometer.
Figure 1 presents the energy dependence of the registration efficiency for the ${ }^{3} \mathrm{He} \mathrm{UCN}$ detector with an $\mathrm{Al}$ window (100 $\mu \mathrm{m}$ thickness) and for the Si detector with a single ${ }^{6} \mathrm{LiF}\left(80 \%{ }^{6} \mathrm{Li}\right.$ enrichment) converter on its surface. Efficiencies of both detectors were compared in the upper part of the spectrum when detectors were in the down position.

The efficiency of the Si detector is better than that of the ${ }^{3} \mathrm{He}$ detector although the boundary velocity of ${ }^{6} \mathrm{LiF}$ ( $80 \%{ }^{6} \mathrm{Li}$ enrichment) is higher than the boundary velocity of the ${ }^{3} \mathrm{He}$ detector $\mathrm{Al}$ window. It is connected with that the absorption coefficient of materials with a high capture cross section is sufficiently bigger for the 


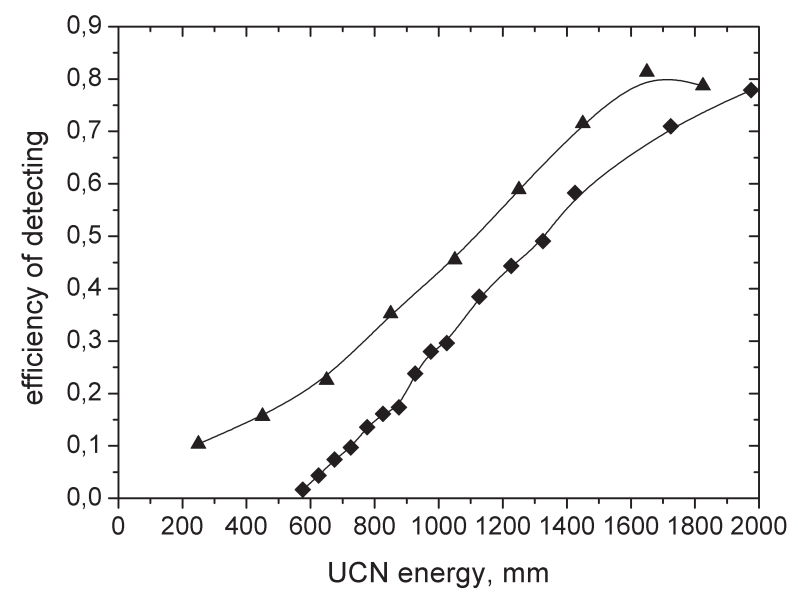

Fig. 1. Energy dependence of registration efficiency: ${ }^{3} \mathrm{He} \mathrm{UCN}$ detector with $\mathrm{Al}$ window $(100 \mu \mathrm{m}) ; \boldsymbol{\Delta} \mathrm{Si}$ detector with ${ }^{6} \mathrm{LiF}(80 \%$ ${ }^{6} \mathrm{Li}$ enrichment) converter on its surface.

low energy region and that the density of coated ${ }^{6} \mathrm{LiF}$ is $30 \%$ lower than the density of a LiF crystal [1].

\section{A Si Sandwich UCN Detector With Two Si Wafers and a ${ }^{6} \mathrm{LiF}$ Converter}

This detector was developed at PNPI and investigated in an experiment at ILL. It allowed one to obtain the fraction of $\alpha$-particles lost in the ${ }^{6} \mathrm{LiF}$ converter by signal summation from the first and the second detectors and application of coincidence and anticoincidence techniques.

The fraction of signals without coincidence is about $28 \%$ and corresponds to events for which the triton was registered and the $\alpha$-particle was absorbed in the ${ }^{6} \mathrm{LiF}$ converter. The thickness of the converter was between $0.6 \mathrm{mg} / \mathrm{cm}^{2}$ and $0.8 \mathrm{mg} / \mathrm{cm}^{2}$.

The results of the measurements allow us to conclude that the detector with one $\mathrm{Si}$ wafer and ${ }^{6} \mathrm{LiF}$ converter looses $14 \%$ of events due to $\alpha$-particle absorption in the ${ }^{6} \mathrm{LiF}$ converter. Thus, the registration efficiency of this detector is about $86 \%$. This conclusion does not contradict with the results of the registration efficiency measurements carried out with the gravitational spectrometer.

The Si sandwich UCN detector is fully efficient for tritons, but decreases the flux of falling UCN with energies of $200 \mathrm{neV}$ to $300 \mathrm{neV}$ by about $20 \%$ to $25 \%$. Thus, the Si sandwich detector has $75 \%$ to $80 \%$ efficiency for UCN with energies of $200 \mathrm{neV}$ to $300 \mathrm{neV}$. It should be used with a vertical guide to accelerate
UCN in the gravitational field, like the ${ }^{3} \mathrm{He}$ detector with an $\mathrm{Al}$ window.

\section{A Si UCN Detector With Polarization Analysis}

The scheme of this detector is presented in Fig. 2. The detector consists of an upper detector for registration of the "up" spin component and a down detector for registration of the "down" spin component. "Up" and "down" polarization components are defined in compliance with the position of the neutron energy levels in the magnetic field. A magnetized ferromagnetic film is placed between the detectors which reflects the "up" spin component and allows the "down" spin component to pass. The down detector is a mosaic of five single detectors $2 \mathrm{~cm} \times 6 \mathrm{~cm}$. The upper detector consists of four single detectors $2 \mathrm{~cm} \times 6 \mathrm{~cm}$ grouped around the neutron guide. The area of the upper detector is $20 \%$ less than the area of the down detector and a part of the reflected neutrons returns to the neutron guide. These neutrons have a chance to return and to be analyzed again.

The analyzing power of the detector can be defined by the polarizing ratio:

$$
R=\frac{N_{\mathrm{d}}-N_{\text {up }}}{N_{\mathrm{d}}+N_{\text {up }}}=P \cdot A,
$$

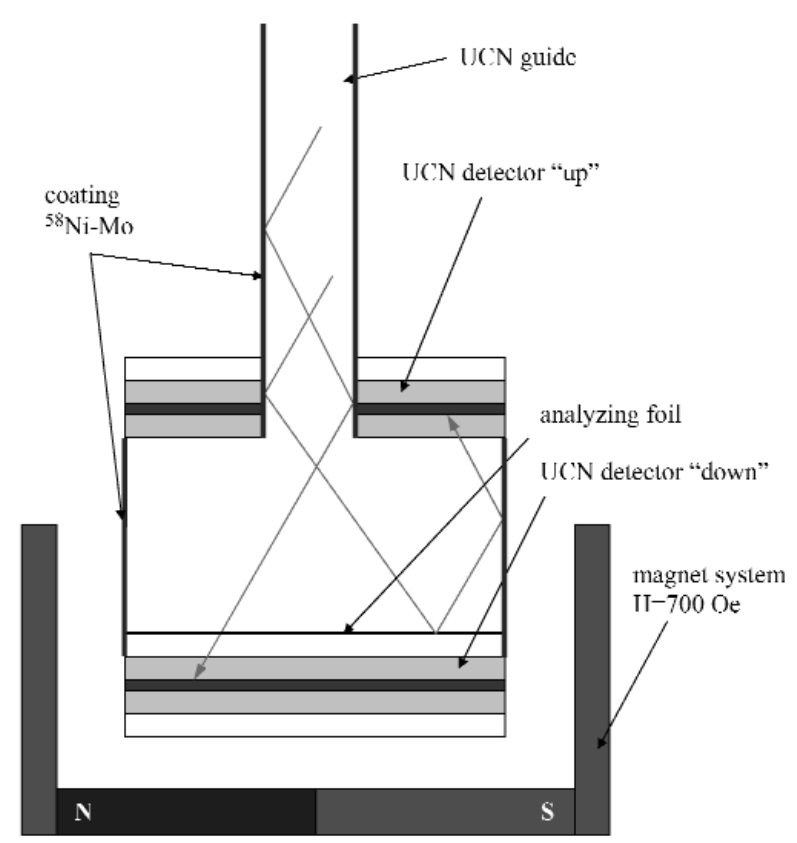

Fig. 2. Scheme of Si UCN detector with polarization analysis. 
where $N_{\text {up }}$ is the count rate of the upper detector, $N_{\mathrm{d}}$ is the count rate of the down detector, $P$ is the UCN polarization, and $A$ is the analyzing power. In the ideal case this ratio is equal to 1 when the flux is completely polarized (the superconducting solenoid-polarizer is switched on) and it is equal to 0 when the flux is unpolarized (the solenoid is switched off). In reality there are several circumstances which disturb these correlations: the registration efficiencies of the detectors are different because of different areas; there is an effect of reflection of UCN from materials which does not depend on the polarization of the neutrons and decreases the efficiency of the polarization analysis; and the analyzing power of the detector depends on the properties of the analyzing ferromagnetic foil. Different materials which could be used as a substrate for thin ferromagnetic films were studied: Al foils (annealed and unannealed) and Si wafers.

Figure 3 shows energy dependence of the polarizing ratio (1) for the flux with the full polarization (solenoid is switched on, $H=4 \mathrm{~T}$ ) and for unpolarized flux (solenoid is switched off, $H=0 \mathrm{~T}$ ). When we change the UCN polarization from 1 to 0 , the polarizing ratio is changed by 0.8 . Overall this result is not bad. The imperfection in splitting of spin components is connected with the effect of UCN reflection from the detector and the substrate of the analyzer. Measurements of the detector count rate ratios with an $\mathrm{Al}$ substrate but without ferromagnetic film showed that the albedo effect is determinative. Using the ratio obtained in these measurements as a correction allows one to reconstruct

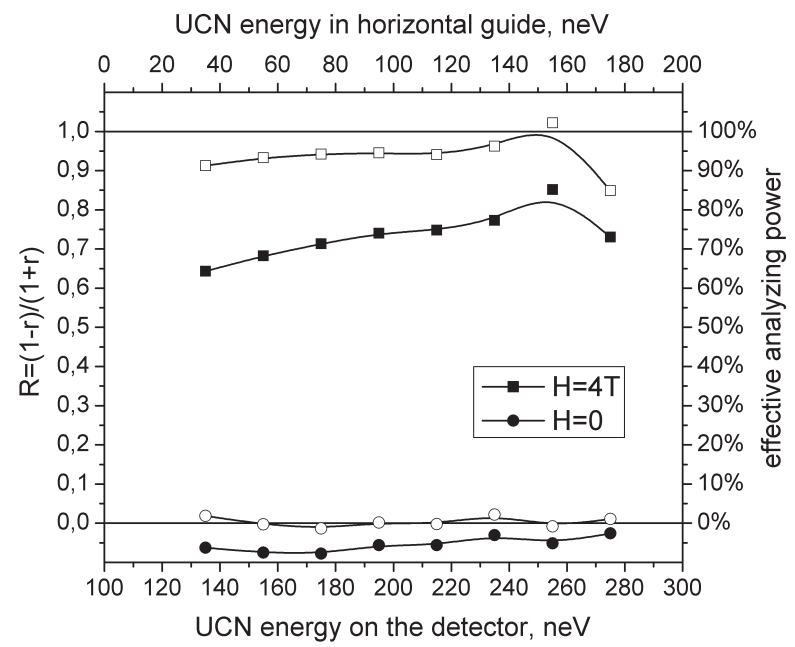

Fig. 3. Energy dependence of polarizing ratio: $\square$ are for flux with full polarization (solenoid is switched on, $H=4 \mathrm{~T}$ ); $\bullet$ are for unpolarized flux (solenoid is switched off, $H=0 \mathrm{~T}$ ). $\square$ and $\circ$ correspond to the values corrected for the albedo effect. almost completely the full polarization value. The empty squares and circles are polarization ratios for polarized and unpolarized flux after correcting for the albedo effect.

In conclusion, the newly developed UCN detector with polarization analysis is very promising, e.g., for an application in the nEDM experiment. Obtained parameters of this detector are sufficient to solve the task, but further improvements are expedient.

\section{Acknowledgments}

The authors are grateful to: K. Kirch and O. Naviliat for useful discussions, Paul Scherrer Institute for the financial support of this work as well as the Russian Foundation of Basic Research for the support under the contracts 02-02-17120 and 04-02-17440, INTAS for the support under the contract 2001-765, the Russian Academy of Science for the support under the Program of Fundamental Research.

\section{References}

[1] J. C. Bates, NIM 150, 261-272 (1978). 